\title{
VARIABILITES MORPHOLOGIQUE ET DU TAUX D'HORMONE DE CROISSANCE DES CIVELLES D'ANGUILLES EUROPEENNES (Anguilla anguilla) DANS L'ESTUAIRE DE LA GIRONDE AU COURS DE LA SAISON 1997-1998
}

\author{
P. LAMBERT (1), M. SBAIHI (2), E. ROCHARD (1), J. MARCHELIDON (2),
} S. DUFOUR (2), P. ELIE (1),

(1) Cemagref, Département Gestion des milieux aquatiques, Unité Ressources aquatiques continentales. 50 avenue de Verdun 33612 CESTAS Cedex - Tél : 33 (0)5 57890800 Fax : $33(0) 557890801$

(2) Muséum National d'Histoire Naturelle Laboratoire de Physiologie, UMR 8572 CNRS. 7, rue Cuvier, 75231 Paris Cedex 05, France - Tél : 33 (0)1 40793612 - Fax : 33 (0)1 40793618

\section{RÉSUMÉ}

L'objectif de ce travail est de compléter la caractérisation morphologique des civelles entrant dans l'estuaire de la Gironde par un paramètre physiologique, le taux d'hormone de croissance. Le flux d'arrivée a été suivi lors de la saison 1997 - 1998. 303 civelles au stade Vb ont été capturées et analysées individuellement. La longueur et la masse des individus diminuent en février, le facteur de condition seulement en mars. Le taux moyen d'hormone de croissance passe de $6 \mathrm{ng} / \mathrm{hyp}$ ophyse en moyenne en décembre à $16 \mathrm{ng} / \mathrm{hyp}$ ophyse en février et mars pour atteindre $20 \mathrm{ng} / \mathrm{hypophyse}$ en avril et mai. L'hypothèse la plus plausible est que cette augmentation du taux d'hormone traduise une détresse physiologique de plus en plus importante des animaux qui arrivent. migration

Mots clef : Anguilla anguilla, civelle, morphologie, hormone de croissance,

\section{MORPHOLOGICAL AND GROWTH HORMONE VARIABILITIES OF EUROPEAN GLASS-EEL (Anguilla anguilla) IN GIRONDE ESTUARY DURING 1997-1998 SEASON}

\section{ABSTRACT}

The aim of this study is to complete the morphological description of Gironde glass eels according to a physiological parameter, the growth hormone. The incoming flux had been observed during the migration season 1997-1998. 303 glass eels of pigmentation stage $\mathrm{Vb}$ were caught and individually analysed. The length and the weight of the glass eels decreased in February, the condition factor only in March. The growth hormone rate increased from an average of $6 \mathrm{ng} /$ pituitary in December to an average of $16 \mathrm{ng} /$ pituitary in February and March and up to an average around $20 \mathrm{ng} / \mathrm{pituitary}$ in April and May. The most credible hypothesis is that the first incoming glass eels show a smaller distress level than the last ones.

Key words : Anguilla anguilla, glass eel, morphology, growth hormone, migration 


\section{INTRODUCTION}

La majeure partie des indicateurs issus de suivis de pêcheries ou de campagnes scientifiques indique actuellement une diminution importante du stock continental de l'anguille européenne (Anguilla anguilla) par rapport à la situation observée à la fin des année 1970 (ANONYME, 1984 ; ANONYME, 1998). L'importance des flux de civelles constitue un indicateur de l'état de santé du stock d'anguille, outil nécessaire dans le processus de gestion à l'échelle européenne. Sa connaissance est un élément utile au niveau de chaque bassin versant pour évaluer les cibles d'échappement de géniteurs. Ce travail s'inscrit dans cette problématique de définition des bases biologiques de la gestion du stock d'anguille en s'attachant à intégrer la caractérisation physiologique des civelles en migration dans l'estuaire de la Gironde.

Depuis les travaux de ELIE (1979) sur la migration estuarienne et sur la succession des stades pigmentaires et leur description, de nombreux travaux ont présenté l'évolution des caractéristiques pigmentaires des civelles en estuaire (CANTRELLE, 1981; GASCUEL, 1987 ; GUERAULT et al., 1992 ; DESAUNAY et al., 1993 ; ELIE et ROCHARD, 1994 ; DESAUNAY et GUERAULT, 1997 ; DE CASAMAJOR, 1998). Ils décrivent tous une mise en place progressive de la pigmentation mélanique des individus. Parallèlement à ces descriptions, des mesures morphologiques concluent à une diminution plus ou moins régulière de la longueur et de la masse. Avec les travaux de MARCHELIDON et al. (1996) qui ont permis le dosage, individu par individu, de l'hormone de croissance (GH pour growth hormone), il est possible d'intégrer une caractéristique endocrinienne dans la description d'un flux de civelles migrantes. Bien qu'elle ne soit pas identifiée par FONTAINE et CALLAMAND (1941) comme jouant un rôle majeur dans la migration des civelles, l'hormone de croissance est impliquée dans plusieurs mécanismes susceptibles d'influer sur le comportement migratoire. En effet, outre son rôle dans la croissance, cette hormone hypophysaire favorise généralement un métabolisme élevé, en particulier dans les situations de détresse physiologique comme le stress ou le jeûne (MARCHELIDON et al., 1996 ; ROUSSEAU et al., 1999). Elle jouerait ainsi un rôle d"hormone de survie" chez les téléostéens (BJORNSSON, 1997 pour une revue). Elle interviendrait également dans d'autres fonctions telles que l'osmorégulation, la fonction immunitaire ou le comportement.

L'objectif de ce travail est donc de visualiser conjointement l'évolution des caractéristiques morphologiques et du taux d'hormone de croissance durant une saison de migration et de mettre en évidence une éventuelle typologie des individus qui entrent en zone estuarienne

\section{MATÉRIEL ET MÉTHODE}

Les prélèvements de civelles ont été réalisés dans le cadre de la surveillance de l'estuaire de la Gironde. Le secteur échantillonné couvre une surface de $27 \mathrm{~km}$ de long sur une dizaine de kilomètres de large. L'échantillonnage biologique et le suivi des paramètres environnementaux sont effectués sur 24 stations réparties dans 4 transects situés respectivement à $31,43,47$ et $51 \mathrm{~km}$ de l'embouchure. Chaque transect comprend 3 stations de surface, une à proximité de chaque rive, la dernière dans l'axe de l'estuaire, et 3 stations de fond avec la même répartition (Figure 1).

Les échantillonnages ont été réalisés durant la saison de migration anadrome 1997-1998 et plus précisément durant les mois de décembre, février, mars, avril et mai (Tableau I). Le protocole d'échantillonnage est similaire à ceux décrits par ELIE et ROCHARD (1994) et par PRONIER et ROCHARD (1998). Les prélèvements sur chaque station durent 5 minutes entre le premier tiers du flot et l'étal de pleine mer. Ils sont en général effectués de jour, sauf ceux du mois de décembre sur les transects 3 et 5 . Le navire est gréé pour réaliser simultanément les prélèvements de fond et de surface. 


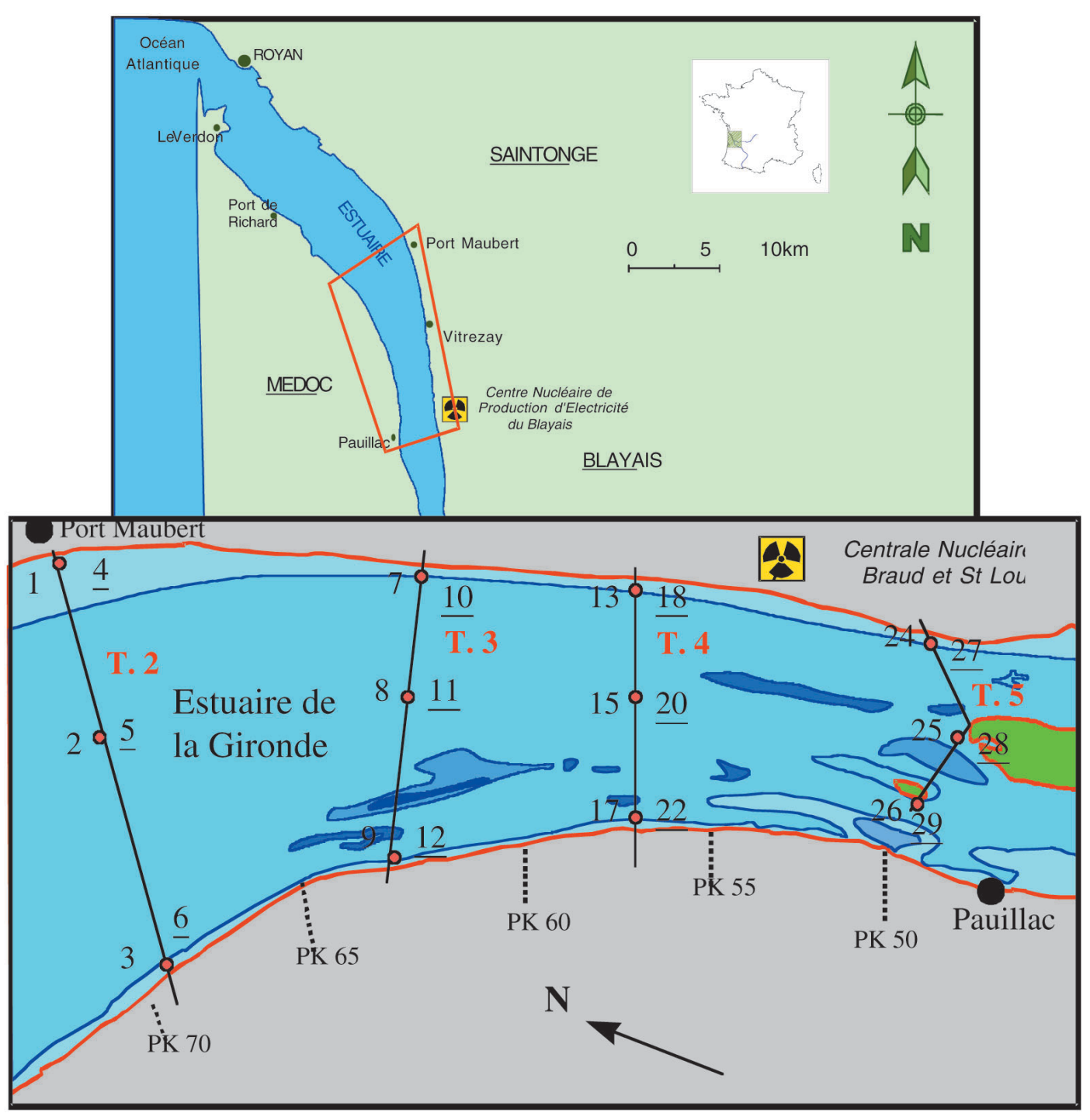

\section{Figure 1}

Localisation des stations d'échantillonnage dans l'estuaire de la Gironde.

\section{Figure 1}

Location of the sampling stations in the Gironde estuary.

Durant l'échantillonnage, il fait face au courant avec une vitesse d'environ 1 nœud par rapport au fond. Les échantillonnages de surface ont été effectués à l'aide de 2 cadres de type «pibalour » portés de part et d'autre du bateau. Leur section est de $4 \mathrm{~m}$ par $1 \mathrm{~m}$. Le maillage étiré est de $18 \mathrm{~mm}$ dans la partie principale de l'engin (ventre et dos) et de $2,8 \mathrm{~mm}$ dans la partie terminale. Les échantillonnages de fond, ont été effectués à l'aide d'un traîneau supra-benthique constitué d'un cadre métallique de 2,0 m sur 1,2 m, maintenu à $0,2 \mathrm{~m}$ du fond par des patins. Le maillage de cet engin est identique à celui des filets utilisés en surface. 
Tableau I :

Calendrier des interventions de terrain durant la saison de migration 1997-1998.

Table I

Agenda of field experiment during migration run 1997-1998.

\begin{tabular}{|c|c|c|c|c|c|c|c|c|}
\hline \multirow[t]{2}{*}{ Mois } & \multirow[t]{2}{*}{ Jour } & \multirow{2}{*}{$\begin{array}{l}\text { Coefficient } \\
\text { de marée }\end{array}$} & \multicolumn{3}{|c|}{ Transect } & \multirow[b]{2}{*}{5} & \multirow{2}{*}{$\begin{array}{c}\text { Température } \\
\text { moyenne } \\
\left(e^{\circ}{ }^{\circ}\right)\end{array}$} & \multirow{2}{*}{$\begin{array}{c}\text { Conductivité } \\
\text { moyenne } \\
\left(\mathrm{en} \mu \mathrm{s} / \mathrm{cm}^{2}\right)\end{array}$} \\
\hline & & & 2 & 3 & 4 & & & \\
\hline Décembre & $\begin{array}{l}15 \\
16\end{array}$ & $\begin{array}{l}94 \\
89 \\
\end{array}$ & $X$ & $x$ & $x$ & $x$ & 6,6 & 5326 \\
\hline Février & $\begin{array}{l}10 \\
11 \\
\end{array}$ & $\begin{array}{l}84 \\
88 \\
\end{array}$ & $x$ & $x$ & $X$ & $X$ & 5,9 & 4846 \\
\hline Mars & $\begin{array}{c}9 \\
10 \\
16 \\
\end{array}$ & $\begin{array}{l}61 \\
72 \\
84 \\
\end{array}$ & $x$ & $x$ & $X$ & $x$ & 10,5 & 2295 \\
\hline Avril & $\begin{array}{l}21 \\
22 \\
\end{array}$ & $\begin{array}{l}49 \\
53 \\
\end{array}$ & $x$ & $x$ & $X$ & $x$ & 11,1 & 2796 \\
\hline Mai & $\begin{array}{l}12 \\
13\end{array}$ & $\begin{array}{l}83 \\
81\end{array}$ & $X$ & $x$ & $x$ & $X$ & 15,0 & 4919 \\
\hline
\end{tabular}

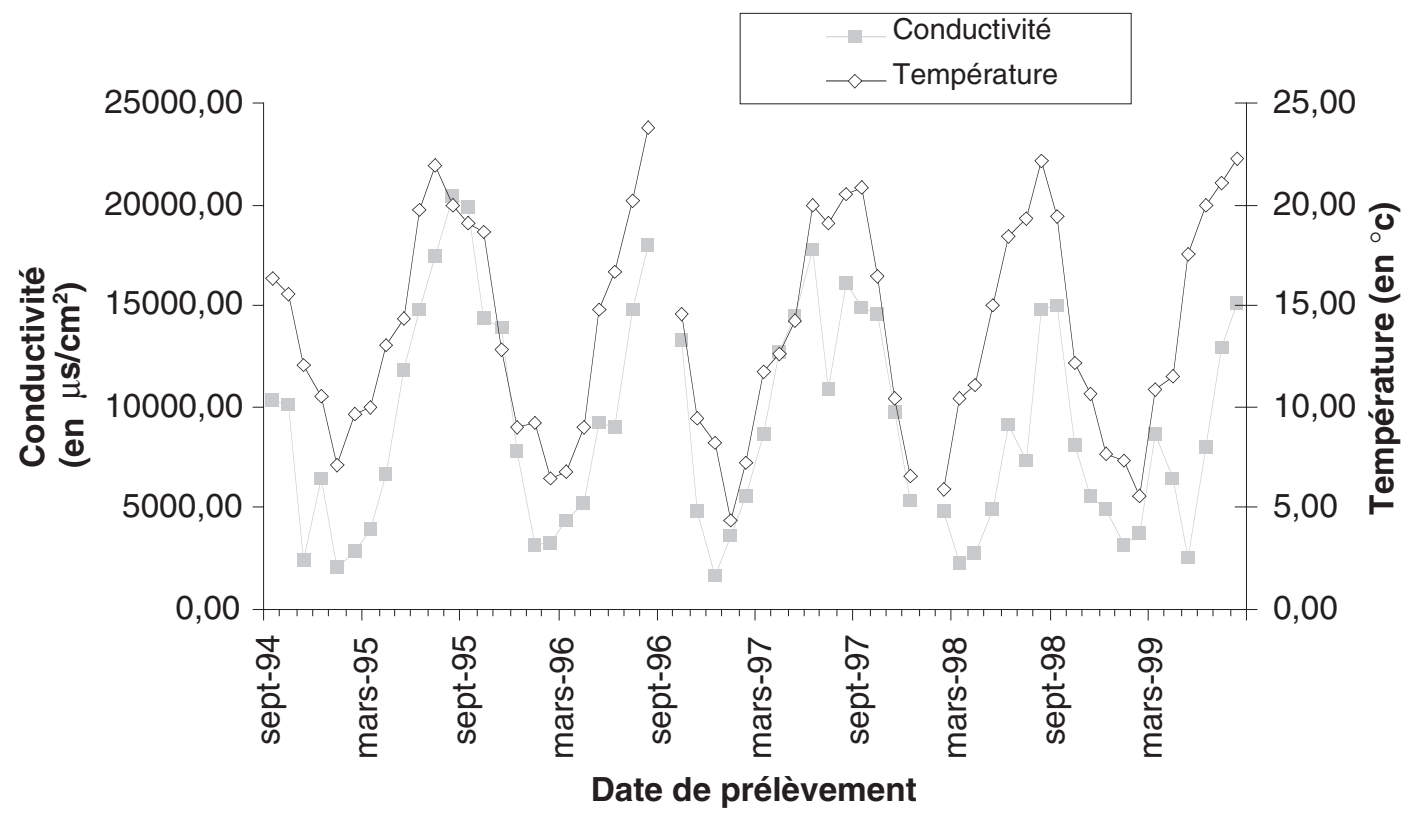

Figure 2 :

Evolution des valeurs mensuelles moyennes de salinité et de la température sur la zone d'étude entre septembre 1994 et août 1999.

Figure 2

Evolution of monthly average salinity and monthly average temperature on the studied zone between September, 1994 and August, 1999. 
Pour chaque station, la température et la conductivité de l'eau sont mesurées avec un SCTmètre de marque YSI. Les conditions environnementales rencontrées entre décembre 1997 et mai 1998 sont comparables à celles enregistrées avant et après cette période (Figure 2). La température est en moyenne de $9,8^{\circ} \mathrm{C}$ ( $\min$. : $5,9^{\circ} \mathrm{C}$, max. : $15,0^{\circ} \mathrm{C}$ ). Pour la conductivité, la moyenne est de $4036 \mu \mathrm{s} / \mathrm{cm}^{2}$ (min. : $2295 \mu \mathrm{s} / \mathrm{cm}^{2}$, max. : $\left.5326 \mu \mathrm{s} / \mathrm{cm}^{2}\right)$.

Les civelles sont triées puis stockées individuellement dans des flacons en plastique. Ces flacons sont ensuite immergés rapidement dans des vapeurs d'azote liquide pour une congélation quasi immédiate à $-276^{\circ} \mathrm{C}$. Le temps entre la fin du prélèvement et la congélation n'excède pas 15 minutes. Les échantillons sont ensuite ramenés au laboratoire et conservés à $-18^{\circ} \mathrm{C}$ avant analyse.

Chaque civelle est mesurée au millimètre près, pesée au millième de gramme et son stade pigmentaire déterminé en se référant aux travaux de ELIE (1979) et ELIE et al. (1982). Elles sont ensuite décapitées. Les individus sont maintenus sur des lits de glace pilée lors des analyses morphologiques afin d'éviter une décongélation avant la décapitation. Tête et corps sont ensuite stockés dans des flacons séparés à $-18^{\circ} \mathrm{C}$.

A partir du broyat de chaque tête de civelle, les teneurs en hormones de croissance sont estimées individuellement suivant la méthodologie définie par MARCHELIDON et al., (1996).

Le facteur de condition allométrique (RICKER, 1980) est calculé par la relation

$$
a=\frac{W}{L^{b}} 10^{6}
$$

où $\mathrm{W}$ représente la masse en $\mathrm{g}$, $\mathrm{L}$ la longueur en $\mathrm{mm}$ et $\mathrm{b}=3,157$ correspond à la pente de la régression linéaire entre le logarithme de la masse et le logarithme de la longueur pour l'ensemble des civelles comme proposé par BOLGER et CONNOLY (1989).

Dans ce travail, seuls les résultats obtenus sur des civelles au stade $\mathrm{Vb}$ sont présentés. Ce stade correspond au stade de colonisation préférentielle des eaux estuariennes (ELIE, 1979 ; CANTRELLE, 1981; ELIE et RIGAUD, 1984 ; GASCUEL, 1987; DESAUNAY et al., 1993, DE CASAMAJOR, 1998). II correspond également au stade le plus fréquent : $88 \%$ des individus capturés pendant la saison 97-98 étaient à ce stade. Pour plus d'information sur la morphologie et le taux d'hormone des autres stades on pourra consulter LAMBERT et al. (1999).

De manière à étudier la variabilité de la taille, de la masse, du facteur de condition et du taux d'hormone de croissance, un modèle linéaire généralisé (procédure GLM de Systat, 1998) basé sur la sommation d'une constante, d'un effet mois, d'un effet profondeur, d'un effet transect et d'un effet rive a été ajusté pour chaque paramètre. Les variables dépendantes ont subi une transformation logarithmique afin de stabiliser leur variance et d'éviter des valeurs négatives. Toutefois, les résultats sont présentés dans leurs échelles naturelles. Pour les effets significatifs, des tests de Bonferroni ont été effectuées pour comparer deux à deux les modalités.

Dans un deuxième temps, afin d'identifier des types de civelles aux caractéristiques voisines, une classification ascendante hiérarchique des individus a été réalisée en se basant sur les valeurs de longueur, de masse, du facteur de condition et du taux d'hormone de croissance. Ces variables ont d'abord subi une transformation logarithmique puis ont été centrées et réduites. La méthode d'agrégation est celle de Ward avec une métrique euclidienne (SYSTAT, 1998). 


\section{RÉSULTATS}

Il convient tout d'abord de noter la proportion importante des civelles au stade $\mathrm{Vb}$ même en fin de période de migration (Tableau II). Un test $d u \chi^{2}(p=0,815)$ ne montre pas de lien entre les mois et la pigmentation ( $\mathrm{Vb}$ ou autre stade). Compte tenu du faible effectif capturé en mai, le traitement statistique n'a été mené que sur les individus pêchés de décembre à avril.

\section{Tableau II :}

Effectifs d'individus capturés en fonction des mois et des stades pigmentaires.

\section{Table II}

Number of caught individuals according to months and pigmentation stages.

\begin{tabular}{lll}
\hline & Vb & Total \\
\hline Décembre & 135 & 145 \\
Février & 96 & 110 \\
Mars & 53 & 64 \\
Avril & 13 & 16 \\
Mai & 6 & 8 \\
Total & 303 & 343 \\
\hline
\end{tabular}

La variance expliquée par les modèles GLM est de $14 \%$ pour la taille, $21 \%$ pour la masse, $14 \%$ pour le coefficient de condition et $40 \%$ pour l'hormone de croissance. Quels que soient les paramètres, les effets transect, rive et profondeur ne sont pas significatifs $(p>0,05)$. Seul l'effet mois s'avère hautement significatif $(p<0,001$ pour les quatre paramètres). La Figure 3 représente l'évolution de la moyenne des moindres carrées et de l'erreur type des différents paramètres en fonction du mois.

La taille moyenne passe de $70 \mathrm{~mm}$ en décembre à $66 \mathrm{~mm}$ en février, puis se stabilise autour de cette valeur les mois suivants (Figure 3a). Statistiquement, le mois de décembre est significativement différent des mois de février et mars $(p<0,001)$.

La masse moyenne diminue depuis décembre jusqu'en avril, passant ainsi de $0,314 \mathrm{~g}$ à $0,234 \mathrm{~g}$ (Figure $3 \mathrm{~b}$ ). Seul, le mois de décembre est significativement différent des autres mois $(p<0,001)$.

Le coefficient de condition montre une diminution plus marquée de février à avril (Figure 3c). II passe ainsi de 0,476 en décembre, à 0,464 en février et à 0,406 en avril. Les valeurs de décembre sont significativement différentes de celles de mars et avril ( $p<0,001$ pour les deux mois), de même pour celles de février par rapport à mars $(p=0,003)$ et par rapport à avril $(p=0,004)$.

Le taux moyen d'hormone de croissance augmente entre décembre et février de $6,4 \mathrm{ng} /$ hypophyse à $16,0 \mathrm{ng} / \mathrm{hyp}$ pophyse, puis en avril où il atteint 19,6 ng/hypophyse (Figure $3 d$ ). Seules les valeurs de décembre diffèrent significativement de celles de février, mars et avril $(p<0,001)$.

Nous avons choisi d'identifier quatre groupes à partir de la classification ascendante hiérarchique (Figure 4). Une analyse descriptive à posteriori permet de caractériser ces groupes (Figure 5). Le premier (A) correspond à des civelles ayant un faible taux d'hormone de croissance, mais une taille, une masse et un indice de condition élevés. Le deuxième $(B)$ regroupe des civelles qui présentent pour les quatre paramètres étudiés des valeurs élevées. Le groupe $C$ rassemble des civelles de taille et de masse plus petites mais avec des facteurs de condition et des taux d'hormone de croissance 
élevés. Le dernier groupe (D) se caractérise par des civelles ayant une taille, une masse et un facteur de condition faible mais un taux d'hormone de croissance élevé.
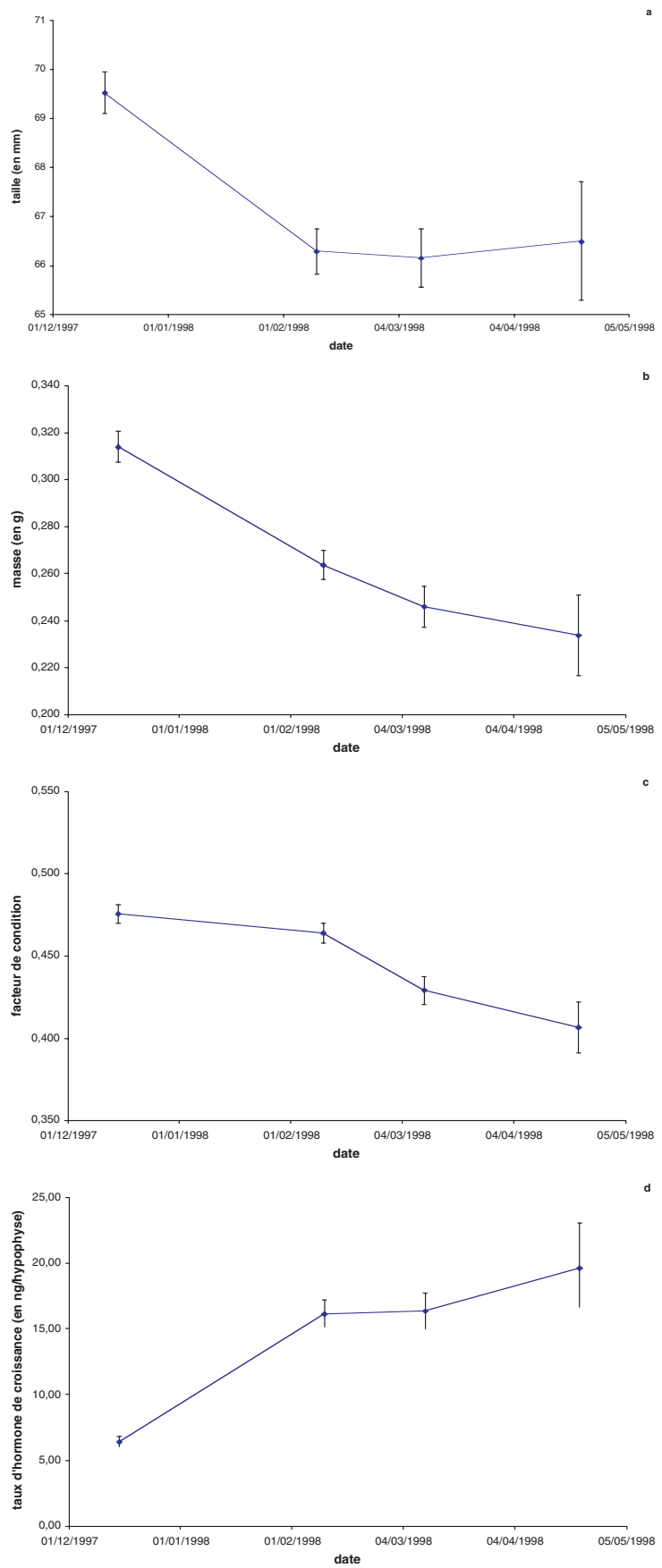

\section{Figure 3}

Evolution de la moyenne des moindres carrées et de l'erreur type pour la taille (a), la masse (b), le facteur de condition (c) et le taux moyen d'hormone de croissance (d) des civelles Vb au cours de la saison de migration 1997-1998.

\section{Figure 3}

Evolution of least square mean and standard error for size ( a ), mass ( b ), condition factor ( c ) and growth hormone rate ( $d$ ) glass eels Vb during the migration season 1997-1998. 


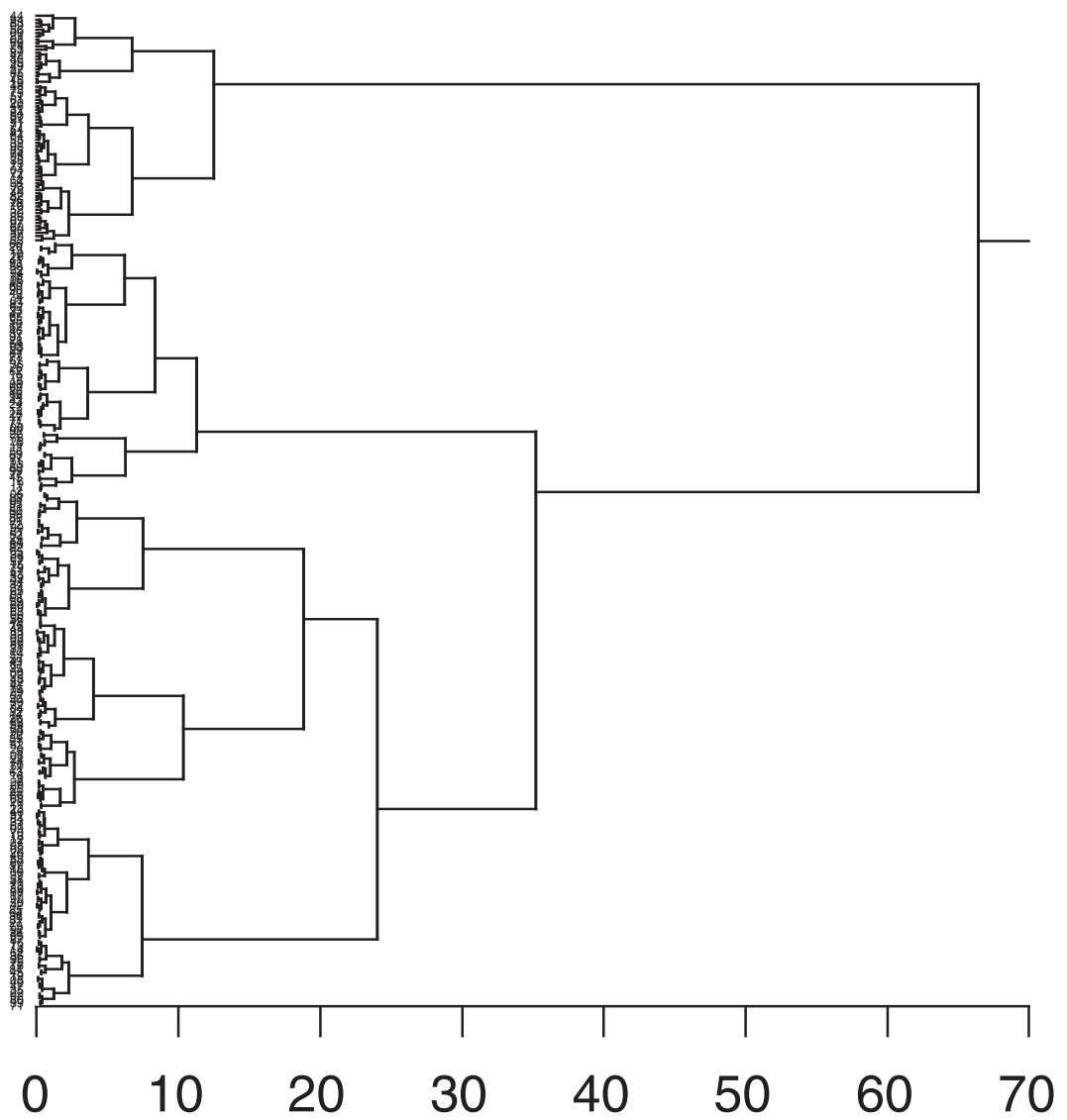

Distance euclidienne

\section{Figure 4}

Dendrogramme issu de la classification ascendante hiérarchique des civelles en fonction de leur taille, de leur masse de leur facteur de condition et de leur taux d'hormone de croissance.

\section{Figure 4}

Hierarchical tree from glass eel clustering according to length, mass, condition and growth hormone rate.

Le groupe $\mathrm{A}$ a été observé principalement en décembre et accessoirement en mars, le groupe $B$ présent en février diminue à partir de mars, le groupe $D$ devient de plus en plus prépondérant en fin de saison de migration (Figure 6).

\section{DISCUSSION}

L'échelle spatiale d'observation des animaux au stade pigmentaire Vb n'a pas permis de montrer de structuration particulière vis à vis des paramètres morphologiques et physiologiques étudiés. Deux hypothèses peuvent être avancées. En premier lieu, l'échelle spatiale d'observation est trop restreinte et l'on peut imaginer qu'une approche le long de l'axe, de la zone marine jusqu'à la zone fluviale aurait probablement permis de mettre en évidence des différences comme dans les travaux de CANTRELLE (1981), CHARLON et BLANC (1982), CIERI (1999) ou DE CASAMAJOR et al. (2001). 

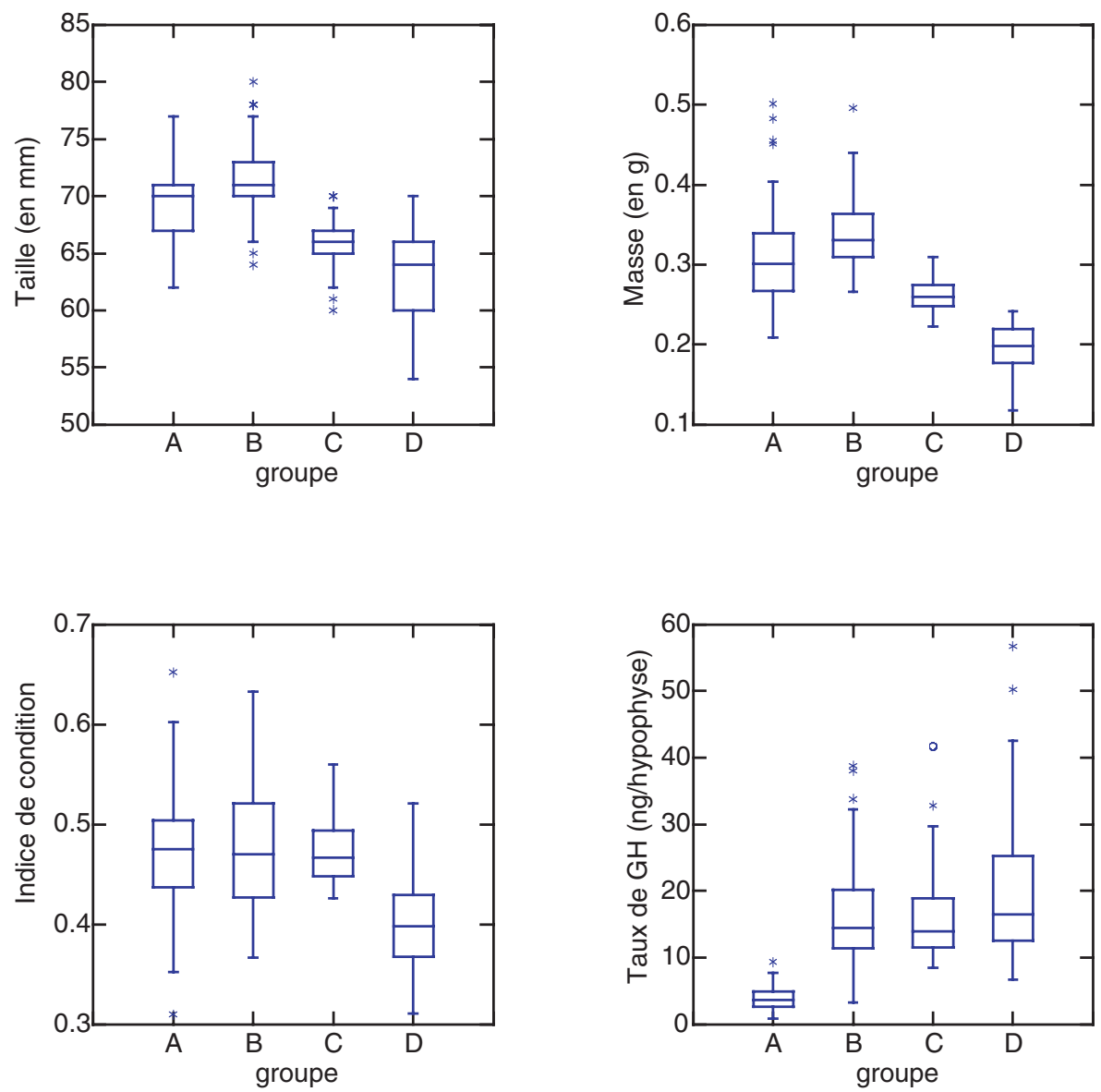

\section{Figure 5}

Diagramme de dispersion pour les groupes issus de la classification ascendante hiérarchique.

\section{Figure 5}

Scatter diagram for the groups from the hierarchical ascending classification.

Deuxièmement, c'est à un stade pigmentaire plus avancé qu'une structuration des flux de migrants apparaîtrait, le stade précoce étudié $(\mathrm{Vb})$ correspondant plutôt à une phase de pénétration passive et non sélective dans les eaux continentales.

La succession des stades pigmentaires observée en 1997-1998 est atypique par rapport à celle déjà observée en Gironde (CANTRELLE, 1981 ; ELIE et ROCHARD, 1994) dans la mesure où la proportion de civelles au stade $\mathrm{Vb}$ ne diminue pas durant notre période d'observation au profit de stades pigmentaires plus avancés.

L'effet sur la taille et le poids de la congélation, imposée par les analyses endocriniennes n'a pu être quantifié dans ce travail. II est clair que les conditions de stockage (frais, formol, alcool ou congélation) et de mesure (individus essuyés ou non) influencent les résultats (ELIE et ROCHARD, 1994). II faut donc, dans les comparaisons avec la littérature existante, ne considérer que l'ordre de grandeur et les tendances d'évolution. 


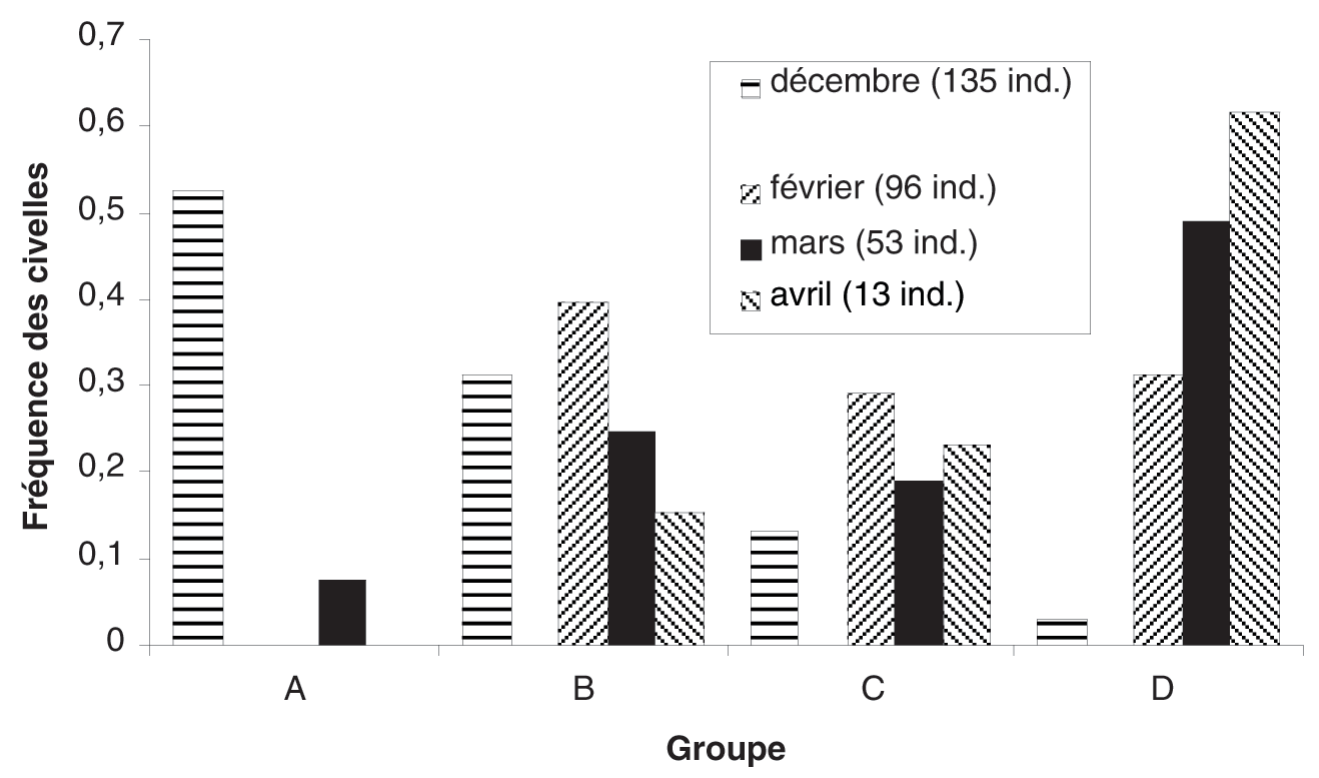

Figure 6

Histogramme de fréquences des différents groupes pour chaque campagne.

\section{Figure 6}

Histogram of the frequencies of the groups for every campaign.

La taille moyenne en début de migration $(70 \mathrm{~mm})$ est comparable avec celle trouvée sur l'Adour, estuaire proche de notre zone d'étude, DE CASAMAJOR, 1998 ; DE CASAMAJOR et al., 2000) mais plus faible que celle citée dans la littérature plus ancienne sur d'autres estuaires français (ELIE, 1979 ; CANTRELLE, 1981 ; CHARLON et BLANC, 1982 ; GASCUEL,1987). Toutefois, DE CASAMAJOR et al. (2001) trouvent sur une saison plus récente (saison 1999-2000) une taille moyenne sur l'Adour de $74,4 \mathrm{~mm}$, plus proche des valeurs historiques.

La diminution de la taille moyenne en fin de migration est moins marquée puisque nous observons une taille de $66 \mathrm{~mm}$, comparable à celle trouvée par ELIE (1979), GASCUEL (1987), DE CASAMAJOR (1998) et DE CASAMAJOR et al. (2001). Elle est inférieure à celle de CANTRELLE (1981). Ces informations sont concordantes avec une tendance à la diminution inter annuelle de la taille des civelles colonisant le lac Den Oever au Pays Bas (DEKKER, 1998)

La diminution de la taille moyenne entre décembre 1997 et février 1998 au cours de la période d'étude est d'environ $1,75 \mathrm{~mm}$ par mois, taux comparable à celui trouvé par DEKKER (1998). La taille moyenne est ensuite stable sur le reste de la saison. ELIE (1979) trouve une relative stagnation seulement à partir de début avril.

La diminution de la masse est quasi régulière de décembre à avril. On trouve une évolution synchrone avec la taille. LECOMTE-FINIGER (1978), ELIE (1979), CANTRELLE (1981), GASCUEL (1987) et DE CASAMAJOR (1998) observent des dimunitions de la taille dans les mêmes gammes de valeurs mais avec des chutes brutales qui interviennent entre janvier et mars suivant les travaux.

Le coefficient de condition moyen par campagne est stable de décembre à février puis diminue jusqu'en avril. On retrouve une évolution similaire chez CANTRELLE (1981) sur la Gironde. GUERAULT et al., 1992 et DESAUNAY et al. 1993 montrent sur 
la Vilaine une augmentation du coefficient de condition de septembre à décembre puis une tendance à la baisse comparable. Même conclusion sur l'Adour chez CHARLON et BLANC (1982). A noter que pour ces références, le facteur de condition est calculé avec un facteur d'allométrie fixé à 3 .

L'évolution des caractéristiques morphologiques observée lors de cette étude est cohérente avec la littérature. Toutefois, si le patron est respecté, le synchronisme des variations est variable.

Le suivi du taux de GH que nous avons réalisé chez des civelles sur une saison de migration constitue une première référence. La comparaison de nos résultats avec ceux obtenus sur des adultes, montrent des valeurs dans des ordres de grandeur de 1 à 1000 : des nanogrammes par hypophyse pour les civelles et des microgrammes par hypophyse pour les adultes (pour revue : DUFOUR, 1996). Le parallèle entre l'évolution au cours de la saison chez les civelles et la différence entre les sub-adultes mâles et femelles (les mâles, à plus faible teneur en hormone de croissance arriveraient alors en début de migration) est donc certainement osé compte tenu du caractère a priori mésogamique du déterminisme du sexe (SOLA et al., 1980 ; WIBERG, 1983)

Chez les Salmonidés, un nombre croissant d'arguments indique que la GH aurait une fonction osmorégulatrice importante en favorisant l'adaptation à l'eau de mer (pour revue : BOEUF, 1993 ; TAKEI, 1993). Cependant, la GH n'aurait pas un rôle osmorégulateur aussi significatif chez les anguilles (pour revue : TAKEI, 1993). En particulier, chez l'anguille japonaise, aucune modification des taux plasmatiques, ni de la clairance de la $\mathrm{GH}$, n'a été observée après transfert d'eau douce en eau de mer (DUAN et HIRANO, 1991).

Dans notre cas, il n'est pas possible d'évoquer le rôle de la GH dans les mécanismes d'osmorégulation pour expliquer les variations de cette hormone. En effet, entre décembre et février, alors que la conductivité est comparable, le taux moyen d'hormone augmente significativement. Symétriquement, alors que la conductivité diminue de février à mars, le taux d'hormone reste stable.

De la même façon, il n'est pas possible de relier les variations du taux moyen d'hormone de croissance avec celles de la température. En effet, quand la température est stable (entre décembre et février), le taux moyen de $\mathrm{GH}$ augmente et réciproquement quand la température augmente (entre février et mars), le taux moyen de $\mathrm{GH}$ ne varie pas.

Par contre, il est intéressant de noter que les civelles au plus fort taux d'hormone de croissance sont aussi les plus petites en taille. Ce paradoxe peut-être levé en rappelant que des taux importants de GH sont liés non seulement à la régulation de la croissance corporelle mais aussi à des situations de forte activité métabolique (mobilisation des réserves énergétiques) dans les cas de détresse physiologique. Cette hypothèse semble être bien adaptée au cas de la civelle qui ne se nourrit pas durant sa métamorphose (ELIE, 1979 ; CHARLON et BLANC, 1983) et qui est obligée de puiser dans ses réserves lipidiques pour migrer en zone estuarienne, même si cela s'effectue de manière essentiellement passive (CHARLON et BLANC, 1983). Nous pouvons donc avancer qu'au fur et à mesure de la saison, les civelles semblent présenter de plus en plus de signes de détresse physiologique.

Ceci ne semble pas pouvoir s'expliquer par une période de jeûne lié à une métamorphose plus longue. En effet, GUERAULT et al. (1992), DESAUNAY et al. (1993) ne montrent pas d'évolution temporelle de la durée entre le début de la métamorphose et l'arrivée dans l'estuaire de la Vilaine. Par contre, cette augmentation de la détresse physiologique pourrait s'expliquer par une différence de réserves lipidiques accumulées durant la traversée océanique (BOËTIUS et BOËTIUS, 1989). Les leptocéphales qui 
migrent durant l'été et qui arrivent les premières (GUERAULT et al., 1992) accumuleraient plus de réserves et entreront en détresse physiologique tardivement. Les civelles de fin de saison, qui ont traversé l'Atlantique en période hivernale, rencontrant des conditions trophiques plus défavorables, ont accumulé moins d'énergie et puiseront plus rapidement dans leurs réserves. Rappellons que la durée de métamorphose comme la saison de traversée transocénique sont basées sur l'estimation de l'âge journalier, méthode qui fait encore l'objet de controverses (LECOMTE-FINNIGER, 1994 ; Mc CLEAVE et al., 1998; TESCH, 1998; CIERI et Mc CLEAVE, 2000; WANG et TZENG, 2000). Cette spéculation sur les réserves lipidiques n'est pas, par ailleurs, confirmée par les mesures de l'état d'amaigrissement où la forte variabilité individuelle du rapport $A D N /$ poids sec observée ne permet pas de dégager de tendance (DE CASAMAJOR et al., 2001).

On peut résumer l'évolution des caractéristiques des civelles au stade pigmentaire Vb durant la saison 1997-1998 comme suit : En décembre, les civelles ont une taille, un poids et un facteur de condition élevés et présentent des taux d'hormone de croissance faible. En février, le taux d'hormone de croissance augmente significativement alors que la taille et le poids diminuent par rapport au mois précédent. Le facteur de condition ne varie pas sensiblement. En mars, le taux d'hormone de croissance la taille et le poids sont comparables aux valeurs de février, seul le facteur de condition présente une diminution significative. En avril, avec des effectifs capturés faible, le taux d'hormone de croissance, le facteur de condition, la taille et le poids ne sont pas significativement différents du mois précédent.

En décembre de cette saison de migration, on peut donc considérer les civelles comme en bonne condition et sans détresse physiologique. En février, les animaux commencent à se trouver dans une situation de détresse physiologique plus grande sans que cela ne se traduise par une modification de leur facteur de condition. A partir de mars, la détresse physiologique des civelles se répercute sur leur "embonpoint". Les quelques civelles du stade $\mathrm{Vb}$ capturées en mai ne confirment pas la tendance exprimée entre décembre et avril. Le facteur de condition remonte à 0,492 en liaison avec une augmentation de la masse des individus $(0,281 \mathrm{~g})$ alors que le taux moyen d'hormone de croissance est comparable à celui du mois précédent (19,33 ng/hypophyse). D'une manière générale, la prudence s'impose dans la généralisation de ce patron d'évolution des paramètres morphologiques et physiologique pour d'autres saisons quand on connaît la variabilité inter - annuelle de ce phénomène (DESAUNAY et al., 1993).

L'approche individuelle permet de préciser la dynamique des caractéristiques des civelles. Le groupe $\mathrm{A}$ correspond visiblement à des civelles en bonne condition. Elles arrivent principalement en début de saison mais également en mars ce qui pourrait correspondre à une nouvelle vague arrivée (ELIE, 1979; GASCUEL, 1987; GUERAULT et al., 1992; ROCHARD,1992; DESAUNAY et al., 1993; DE CASAMAJOR, 1998, DE CASAMAJOR et al., 2000). Le groupe D est associé à des individus en plus mauvaise condition (petite taille, faible facteur de condition, taux de GH élevé). Ils sont observés pendant toute la saison mais deviennent prépondérants en mars et avril. Les civelles des groupes $B$ et $C$ peuvent être considérées comme dans des situations intermédiaires, leur taux d'hormone est déjà élevé, les individus du premier groupe étant plus petits et plus maigres. Elles sont présentes de décembre à avril. Plus qu'une succession de types nous avons affaire à un mélange d'animaux en plus ou moins bonne condition. II est donc difficile de faire la part entre les deux mécanismes, non exclusifs, d'évolution des caractéristiques morphologiques proposé par ELIE (1979), soit une diminution de la taille et de la masse des animaux arrivant dans l'estuaire (liée à des variations des conditions de migration des larves leptocéphales (GUERAULT et al., 1992)), soit une diminution individuelle de la taille et du poids des civelles séjournant en estuaire, conséquence de l'arrêt de la nutrition et des processus de métamorphose. 
Toutefois, nos analyses sont concordantes pour montrer que les animaux au stade $\mathrm{Vb}$ en début de migration sont en meilleure condition qu'en fin. II est pour autant impossible de conclure que les civelles de début de migration ont plus de chance de participer à la colonisation du bassin versant par rapport à celles de fin de saison. Au contraire, GUERAULT et al. (1992) estiment que les civelles qui arrivent en début de saison en estuaire, rencontrant des situations environnementales défavorables (faible température de l'eau, support trophique limité pour la reprise d'alimentation) ont une faible chance de survie jusqu'au stade "anguillette", alors que celles qui arrivent au printemps dans un milieu plus "accueillant", qui se pigmentent vite du fait des plus fortes températures, qui s'alimentent sans délai et qui colonisent les eaux douces, sont les vraies recrues pour le bassin versant. Cette position est peut-être excessive, il y a plus certainement un équilibre entre des individus du début de migration en meilleure forme mais qui vont rencontrer des conditions environnementales difficiles et des animaux plus faibles en fin de saison qui vont rapidement profiter des conditions favorables à leur croissance. En conclusion cette étude n'apporte pas de justification pour protéger plus les animaux arrivant en début de migration qu'en fin ou de faire reposer la colonisation sur une partie seulement des animaux (après la fermeture de la pêche).

Enfin il est nécessaire pour dépasser les conclusions ponctuelles sur une saison de migration de mettre en place des réseaux pérennes de mesures des paramètres biologiques avec des méthodes standardisées. Des travaux en structures expérimentales devraient également être menés pour préciser les cinétiques d'évolution des caractéristiques morphologiques mais surtout du taux d'hormone de croissance. Ils permettraient en effet de valider les hypothèses d'influence de la température, de la salinité et de la diminution des réserves énergétiques avancées dans ce travail de terrain. Ces expériences pourraient même estimer les conséquences de ces cinétiques sur le taux de survie.

\section{REMERCIEMENTS}

Cette étude a fait l'objet d'un financement Ministère de l'Aménagement du Territoire et l'Environnement - Groupement d'intérêt scientifique sur les poissons amphihalins (GRISAM).

\section{BIBLIOGRAPHIE}

ANONYME, 1984. Rapport de synthèse et programme quinquennal. Groupe national anguille, $60 \mathrm{p}$.

ANONYME, 1998. Extract about eel of the report of the Advisory Committee on Fishery Management, n¹1, may 1998.

BJORNSSON B. Th. 1997. The biology of salmon growth hormone: from daylight to dominance. Fish Physiol. Biochem., 17, 9-24.

BOËTIUS I., BOËTIUS J., 1989. Ascending elvers, Anguilla anguilla, from five Europeans localities. Analyses of pigmentation stages, conditions, chemical composition and energy reserves. Dana, 7, 1-12.

BOEUF G., 1993. Salmonid smolting: a pre-adaptation to the oceanic environment. In: RANKIN J.C., JENSEN F.B., eds, Fish ecophysiology. Chapman et Hall, Publ., London. 105-135.

BOLGER T., CONNOLY P.L., 1989. The selection of suitable indices for the measurement and analysis of fish condition. J.Fish. Biol., 34, 171-182.

CANTRELLE I., 1981. Etude de la migration et de la pêche des civelles Anguilla anguilla L. 1758 dans l'estuaire de la Gironde, Thèse de doctorat de $3^{\text {ème }}$ cycle. Université de Paris VI, $237 \mathrm{p}$. 
CHARLON N., BLANC J.M., 1982. Etude des civelles d'Anguilla anguilla L. dans la région du bassin de l'Adour. 1- Caractéristiques biométriques de longueur et de poids en fonction de la pigmentation. Arch. Hydrobiol., 93 (2), 238-255.

CHARLON N., BLANC J.M., 1983. Etude des civelles d'Anguilla anguilla L. dans la région du bassin de l'Adour. 2- Régime alimentaire et variations de quelques caractéristiques biométriques en cours de migration. Arch. Hydrobiol., 98 (2), 240-249.

CIERI M.D., 1999. Migrations, growth, and early life history of the American eel (Anguilla rostrata). University of Maine, PhD thesis. $117 \mathrm{p}$.

CIERI M.D., McCLEAVE J.D., 2000. Discrepancies between otoliths of larvae and juveniles of the American eel: is something fishy happening at metamorphosis? J. Fish. Biol., 57 (5), 1189-1198.

DE CASAMAJOR M.N., 1998. Comportement migratoire de la civelle d'anguille (Anguilla anguilla L.) dans l'estuaire de l'Adour en fonction de la variabilité des conditions environnementales. Thèse de doctorat, Université de Pau et des Pays de l'Adour. $138 \mathrm{p}+$ annexes.

DE CASAMAJOR M.N., PROUZET P., LAZURE P., 2000. Identification des flux de civelles (Anguilla anguilla) à partir des relations d'allosome en fonction des conditions hydrodynamiques de l'estuaire de l'Adour. Aquat. Living Resour., 13, 411-420.

DE CASAMAJOR M.N., LECOMTE-FINIGER R.,., PROUZET P., 2001. Détermination de l'état d'amaigrissement des civelles (Anguilla anguilla) en migration en zone côtière et estuarienne. C.R. Acad. Sci. Paris, Sciences de la vie / Life sciences, 324, 345-353.

DEKKER W., 1998. Long-term trends in the glass eels immigrating at Den Oever, The Netherlands. Bull. Fr. Pêche et Pisc., 349, 199-214.

DESAUNAY Y., GUERAULT D., 1997. Seasonal and long-term changes in biometrics of eel larvae: a possible relationship between recruitment variation and North Atlantic ecosystem productivity. J. Fish Biol., 51 (supp A), 317-339.

DESAUNAY Y., GUERAULT D., LECOMTE-FINIGER R., 1993. Variation of the oceanic larval migration of Anguilla anguilla (L.) glass eels from a two years study in the Vilaine estuary (France). $8^{\text {th }}$ session of the EIFAC Working party on eel, $7 \mathrm{p}$.

DUAN C., HIRANO T., 1991. Plasma kinetics of growth hormone in the Japanese eel, Anguilla japonica. Aquaculture, 95, 179-188.

DUFOUR S. 1996. Un exemple de cycle reproducteur sous la dépendance de l'environnement : le cas de l'anguille. C.R. Acad. Agric. Fr., 82, 17-26.

ELIE P., 1979. Contribution à l'étude des montées de civelles d'Anguilla anguilla L., dans l'estuaire de la Loire : Pêche écophysiologie et élevage. Thèse de Doctorat de $3^{\text {eme }}$ cycle, Université de Rennes I, 383 p.

ELIE P., LECOMTE-FINIGER R., CANTRELLE I.,CHARLON N., 1982. Définition des limites des différents stades pigmentaires durant la phase civelle d'Anguilla anguilla L. (Poisson téléostéen anguilliforme). Vie Milieu, 32 (3), 149-157.

ELIE P., RIGAUD C., 1984. Etude de la population d'anguilles de l'estuaire et du bassin versant de la Vilaine : pêche, biologie, écologie. Examen particulier de l'impact du barrage d'Arzal sur la migration anadrome (civelle). Tome I, II et III. Rapport CEMAGREF de Bordeaux-Faculté des sciences de Rennes-URFDAAPP, 350 p.

ELIE P. et ROCHARD E., 1994. Migration des civelles d'anguilles (Anguilla anguilla L.) dans les estuaires, modalité du phénomène et caractéristiques des individus. Bull. Fr. Pêche et Pisc., 35, 81-98.

FONTAINE M., CALLAMAND O., 1941. Sur I'hydrotropisme des civelles. Bull. Inst. Océanogr. Monaco, 811, 1-6.

GASCUEL D., 1987. La civelle d'anguille dans l'estuaire de la Sèvre Niortaise, biologie, écologie, exploitation. ENSA Rennes. 4 (1), 353 p.

GUERAULT D., LECOMTE FINIGER R., DESAUNAY Y., BIAGIANTI-RISBOURG S., BEILLOIS P., GRELLIER P., 1992. Glass eels arrivals in the Vilaine estuary (Northen Bay of Biscay) in 1990: Demographic features and early life history. Irish. Fish. Invest. (A. Freshwater.), $\mathrm{n}^{\circ}$ 36, 5-14. 
LAMBERT P., DUFOUR S., ROCHARD E., SBAIHI M., ELIE J., MARCHELIDON J., 1999. Variabilité morphologique et physiologique des civelles d'anguilles dans l'estuaire de la Gironde. Rapport d'étude GRISAM Cemagref Museum. 22 p.

LECOMTE-FINIGER R., 1978. Contribution à l'étude biologique et écologique des civelles (Anguilla anguilla) entrant dans un étang méditerranéen. Thèse de doctorat de troisième cycle, Université Paris VI. $66 \mathrm{p}$.

LECOMTE-FINIGER R., 1994. Contribution de l'otolitholimétrie à l'étude de la dynamique de la migration larvaire de l'anguille européenne Anguilla anguilla. Bull. Fr. Pêche et Pisc., 335, 17-32.

MARCHELIDON J., SCHMITZ M., HOUDEBINE L.M., VIDAL B., LE BELLE N., DUFOUR S. 1996. Development of a radioimmunoassay for European eel growth hormone: an application to the study of silvering and experimental fasting. Gen. Comp. Endocrinol. 102, 360-369.

Mc CLEAVE J.D., BRICKLEY P.J., O'BRIEN K.M., KISTNER D.A., WONG M.W., GALLAGHER M., WATSON S.M., 1998. Do leptocephali of the European eel swim to reach continental waters? Status of the question. J. Mar. Biol. Ass., 78. 285-306.

PRONIER O., ROCHARD E., 1998. Fonctionnement d'une population d'éperlan (Osmerus eperlanus, osmériformes osmeridae) située en limite méridionale de son aire de répartition, influence de la température. Bull. Fr. Pêche et Pisc., 350-351, 479-497.

ROCHARD E., 1992. Mise au point d'une méthode de suivi de l'abondance des amphihalins dans le système fluvio-estuarien de la Gironde, application à l'étude écobiologique de l'esturgeon Acipenser sturio. Thèse de doctorat, Université de Rennes I, $315 \mathrm{p}$.

ROUSSEAU K., LE BELLE N., MARCHELIDON J., DUFOUR S. 1999. Evidence that corticotropin-releasing hormone acts as a growth hormone-releasing factor in a primitive teleost, the European eel (Anguilla anguilla). J. Neuroendocrinology. 11, 385-392.

RICKER W.E., 1980. Calcul et interprétation des statistiques biologiques des populations de poissons. Bull. Fish. Res. Board Can., 191F, 409 p.

SOLA L., GENTILI G., CATAUDELLA S., 1980. Eel chromosomes: cytotaxonomical interrelationships and sex chromosomes. Copeia, 1980, 911-913.

SYSTAT, 1998. Systat 8.0 Statistics. SPSS Chicago Inc.1086 p.

TAKEI Y., 1993. Role of peptide hormones in fish osmoregulation. In: RANKIN J.C., JENSEN F.B., eds, Fish ecophysiology. Chapman et Hall, Publ., London. 136-160.

TESCH F.W., 1998. Age and growth rates of North Atlantic eel larvae (Anguilla spp), based on published length data. Helgoländer Meeresunters, 52, 75-83.

WANG C.H., TZENG W.N., 2000. The timing of metamorphosis and growth rates of American and European eel leptocephali: A mechanism of larval segregative migration. Fish. Res., 46, 191-205.

WIBERG U.H., 1983. Sex determination in the European eel (Anguilla anguilla, L.). Cytogenetic and Cell Genetics, 36, 589-598. 
